\title{
The Effectiveness of $0.2 \%$ Chlorhexidine Gel on Early Wound Healing after Tooth Extraction: A Randomized Controlled Trial
}

\author{
Amaliya Amaliya ${ }^{1}$ Rika Ramadhanti ${ }^{2}$ Indra Hadikrishna ${ }^{3}$ Tantry Maulina ${ }^{3}$ \\ ${ }^{1}$ Department of Periodontology, Faculty of Dentistry, Universitas \\ Padjadjaran, West Java, Indonesia \\ 2 Faculty of Dentistry, Universitas Padjadjaran, West Java, Indonesia \\ ${ }^{3}$ Department of Oral Surgery, Faculty of Dentistry, Universitas \\ Padjadjaran, West Java, Indonesia

\begin{abstract}
Address for correspondence Amaliya Amaliya, DDS, MSc, Department of Periodontology, Faculty of Dentistry, Universitas Padjadjaran, Jalan Sekeloa Selatan no. 1 Bandung 40132, West Java,
\end{abstract} \\ Indonesia (e-mail: amaliya@fkg.unpad.ac.id). \\ Eur J Dent 2022;16:688-694.
}

\begin{abstract}
Keywords

- chlorhexidine gel

- wound healing

- tooth extraction
\end{abstract}

Objective This study aimed to evaluate the effect of $0.2 \%$ chlorhexidine (CHX) gel on wound healing after tooth extraction.

Materials and Methods A single blind, randomized controlled trial was performed recruiting 32 participants who underwent dental extractions. Patients were randomly allocated for $\mathrm{CHX}$ group or placebo group. The primary outcomes were wound closure measured with calipers and healings were assessed by Landry et al index after 7 days of topical application of allocated gels on extraction sites.

Results The wound closures were greater in $\mathrm{CHX}$ group compared with placebo group and healing scores were correlated with the use of $\mathrm{CHX}$ gel ( $p$-value $<0.05)$.

Conclusion In a population of healthy nonsmoker adults, application of $0.2 \% \mathrm{CHX}$ gel twice a day for 7 days after tooth extraction has a beneficial effect on wound healing.

\section{Introduction}

Tooth extraction is a very common procedure that dentists perform every single day. Postoperative complication generally do not occur; however, occasionally delayed wound healing may arise even in normal healthy patients. The most frequent postextraction complications documented were infection, prolonged bleeding, swelling, as well as dry socket. ${ }^{1}$ In addition, patient may experience pain even after a simple uncomplicated tooth extraction. ${ }^{2,3}$

Oral healing is slower and delayed compare with dermal repair. ${ }^{4}$ Unlike skin surface, oral environment cannot be sterilized from oral bacteria or plaque formation, leading to persistent environmental challenge for the oral wound. Infection is one of the significant causes of delayed wound healing; therefore, early period of healing after tooth extraction must be facilitated and protected from infection or any condition inhibiting healing and repair. ${ }^{5}$

After the procedure is performed, the site of tooth extraction may serve as a niche for bacterial plaque formation, due to the fact that patients cannot maintain good oral hygiene. ${ }^{6}$ Patients may have hesitation to brush the wound site due to the pain or discomfort and may be instructed by dentist to avoid the brush to avoid hitting the extraction socket. To inhibit bacterial plaque accumulation, several interventions have been incorporated in postextraction procedures such as administration of antibiotics, mouthwashes, or topical medications, thereby preventing infection and chronicity of the wound. ${ }^{7,8}$

Unfortunately, there is emerging situation of antimicrobial resistances caused by antimicrobial abuse, especially from systemic antibiotic use. To overcome this problem, published online January 11, 2022
DOI https://doi.org/ 10.1055/s-0041-1739544. ISSN 1305-7456.

\section{(c) 2022. The Author(s).}

This is an open access article published by Thieme under the terms of the Creative Commons Attribution License, permitting unrestricted use, distribution, and reproduction so long as the original work is properly cited. (https://creativecommons.org/licenses/by/4.0/)

Thieme Medical and Scientific Publishers Pvt. Ltd., A-12, 2nd Floor, Sector 2, Noida-201301 UP, India 
topical antimicrobials in wound therapy are increasing in use..$^{9-11}$ In this method of therapy, topical antimicrobials are directly applied to the wound, resulting in a high concentration at the wound site, low systemic side effects, and a low incidence of antimicrobial resistance. One of topical antimicrobial agents recently available in the market is chlorhexidine (CHX) digluconate. Over a period of 40 years, CHX is widely used in dentistry and considered as an excellent antiplaque agent, due to its high substantivity and broad antimicrobial spectrum. Several forms of CHX have been prepared, that is, mouthwash, spray, chip, cement, varnish, dentifrice, and gel. ${ }^{12,13}$ It has been found to have superior antimicrobial activity compared with other active agents. ${ }^{14}$ Nevertheless, disadvantages remain prominent, it was found that CHX in the form of mouthwash showed the most common side effect of tooth restoration and tongue staining. ${ }^{15,16}$ In addition, there is some evidence that regular and frequent application of CHX mouth rinses may temporarily impair the taste sensation, and promotes supragingival calculus formation and desquamative lesion of oral mucous. ${ }^{17}$ Other disadvantage that may occur that is rinsing 24 hours after tooth extraction may dislodge blood clot that forms in the socket; therefore, a method of administration of $\mathrm{CHX}$ that may enhance and facilitate wound healing after tooth extraction is needed. ${ }^{18}$

Topically applied CHX gel (Perio-Kin) has been proven to enhance wound healing in rats both at the clinical and histological levels without any adverse effect. ${ }^{19}$ The use of the gel preparation had also been shown to reduce the incidence of dry sockets after third molar extraction; nevertheless, the method of treatment could only be performed by the surgeon. ${ }^{20}$ The present study aimed to evaluate the effect of $0.2 \% \mathrm{CHX}$ gel on early wound healing after tooth extraction of mandibular first molar, applied topically on the top of the wound site by the patients.

\section{Materials and Methods}

The study design was a single blind, randomized controlled trial (RCT). The period of the trial was from August to December 2019, and the study protocol was registered in February 2021 at UMIN clinical trial registry with clinical trial registration number UMIN000043357. The participants consisted of 32 healthy nonsmokers who were about to undergo unilateral extraction of mandibular first molar at the Oral Minor Surgery Outpatients Ward of Dental Hospital, Universitas Padjadjaran, Bandung, West Java, Indonesia. Sample size calculation was performed by using the following formula: $(r-1)(t-1) \geq 15$, where $t$ is the number of groups and $r$ is the number of samples.

Therefore, the number of subjects was 16 participants for each group.

Prior to the start of the study, ethical clearance was obtained from the Research Ethics Committee Faculty of Medicine Universitas Padjadjaran, Bandung, Indonesia (1497/UN6.KEP/EC/2018). Every procedure and ethical aspect of the current research has been conducted in full accordance with the World Medical Association's Declara- tion of Helsinki, and all participants gave their consent for their participation in the current study. Inclusion criteria of eligible participants were as follows: (1) patients who underwent tooth extraction of mandibular first molar, (2) aged 18 to 50 years with American Society of Anesthesiologists (ASA) physical status I, (3) did not have the medical history of being allergic to $\mathrm{CHX}$ and mefenamic acid, (4) was not consuming any other antibiotic, analgesic or anti-inflammation drug(s) at least 7 days prior to the procedure, (5) lack of infection at the tooth indicated for extraction 3 days prior to the extraction procedure, and (6) absence of any pathology at the area of the tooth indicated for extraction and neighboring teeth. Participants with medically compromised condition, being pregnant, or with tooth requires surgical extraction were excluded from the study.

Participants who fulfilled the inclusion criteria were then divided randomly into one of the following groups: the test group that received topical administration of $0.2 \% \mathrm{CHX}$ digluconate gel (Perio-kin, Laboratorios Kin S.A., Barcelona, Spain) and $500 \mathrm{mg}$ of mefenamic acid or the control group that received topical administration of placebo gel (Carboxymethyl Cellulose Sodium, glycerin, and aquadest) and $500 \mathrm{mg}$ of mefenamic acid. Randomization was performed by taking a closed envelope containing the name of the group to which the participant was assigned to. The field researcher (T.M.) was responsible for this procedure and supervised each randomization procedure. After the participant was assigned to a group, the field researcher made the necessary record in confidential in the research database.

All participants underwent dental extraction under local anesthesia at the Oral Minor Surgery Outpatients Ward of Dental Hospital, Universitas Padjadjaran, Bandung West Java, Indonesia. The procedures adhered to the standard pre- and postoperative extraction protocols. Each removal of the mandibular first molar procedure was conducted by using $2 \mathrm{~mL}$ of lidocaine $\mathrm{HCl}$ with epinephrine as the local anesthetic and direct inferior alveolar nerve block as the injection technique. Mandibular first molars were extracted using simple extraction with close method, with No. 17 or No. 23 forceps. No. 17 forceps was seated as far apically as possible. Luxation of the molar was initiated with a buccal movement and then to the lingual. Subsequently, molar was delivered in the bucco-occlusal direction. No tooth sectionings were done during all procedures. No sutures have been applied to obtain wound healing by secondary intention. All the patients received postoperative advice on good oral hygiene and information on how to apply the topical gel. All extraction procedures were performed by a certified dental surgeon (I.H.) who was not informed about the group of which the participant was assigned to. All the patients were instructed to apply the assigned gel with cotton applicators onto the extraction wound site two times (every 12 hours) for 7 days, and take the mefenamic acid pill if necessary.

Assessments of wound diameter were performed immediately after extraction and 7 days after procedure, in buccolingual and mesio-lingual widths using a vernier caliper. Healing was assessed using the standardized index by 
Table 1 Characteristics of participants

\begin{tabular}{|l|l|l|l|}
\hline Variable & CHX & Placebo & \multirow{2}{*}{$p$-Value } \\
\cline { 2 - 3 } & $\boldsymbol{N}=16$ & $N=16$ & \\
\hline Age $(\mathrm{y} \pm \mathrm{SD})$ & $26.06 \pm 9.45$ & $26.13 \pm 7.78$ & 0.481 \\
\hline Sex (male/female) & $7 / 9$ & $4 / 12$ & 0.457 \\
\hline
\end{tabular}

Abbreviations: CHX, chlorhexidine; SD, standard deviation.

Landry et al (1988). ${ }^{21}$ Calculation was done by measuring the wound diameter differences between pre-extraction (Day 1) and postextraction (Day 7) assessments.

All measurements were performed under the same conditions by one calibrated examiner (R.R.) who was not informed about the group of which the participant was assigned to. Reproducibility measurements in 20 other postextracted patients, 8 hours apart, showed an intraclass correlation coefficient of 0.93 and 0.90 for wound diameter and healing index measurements, respectively.
All of the data were subjected for normality test (Lilliefors' test). For wound diameter analysis, pair $t$-test was used since it was normally distributed. Due to the fact that the data obtained from healing index were not normally distributed, Kendall's rank correlation (Kendall's tau) was employed to measure the association. Statistical significance was identified by setting the $p$-value $\leq 0.05$. Statistical analysis was performed using SPSS version 23 from IBM, United States.

\section{Results}

A total of 32 subjects (16 in test group and 16 in control group) completed the study. The phases of parallel RCT are shown in the Consolidated Standards of Reporting Trials (CONSORT) flow diagram (-Fig. 1). The mean age of the 32 subjects was 26.09 ( \pm 8.51 ) years without significant differences between the groups and ranged in all the two groups between 19 and 46 years. The study population consisted of 11 males and 21 females which was balanced distributed

\section{CONSORT 2010 Flow Diagram}

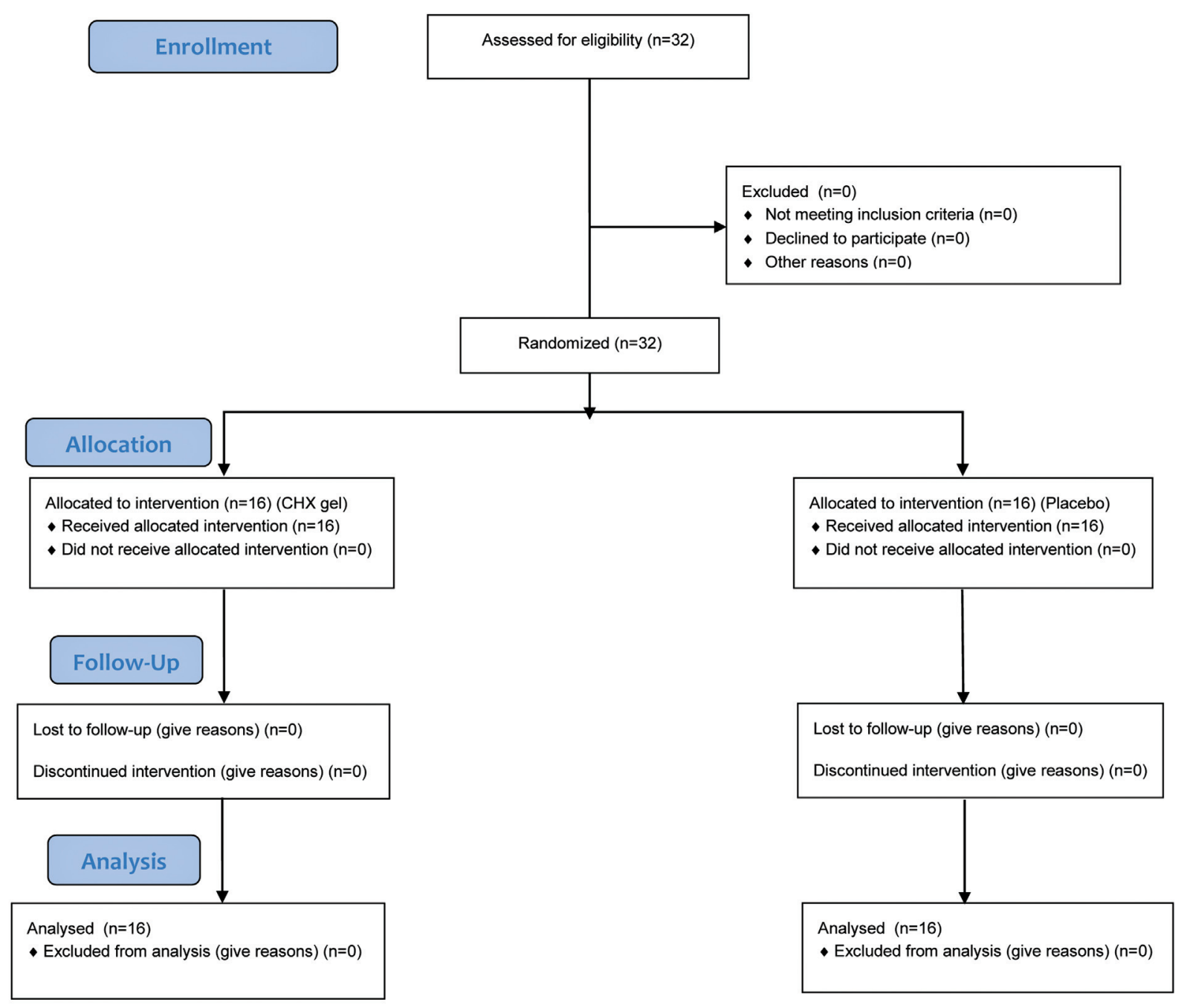

Fig. 1 Consolidated Standards of Reporting Trials (CONSORT) flow of participants. 


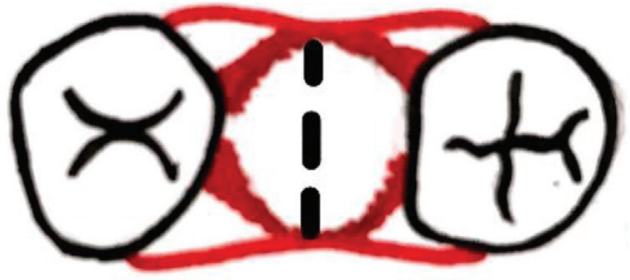

A

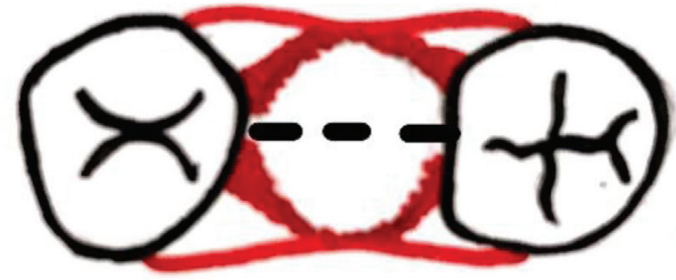

B

Fig. 2 Wound diameter in buccolingual (A) and mesiolingual (B) direction.

over the two study groups ( $\boldsymbol{- T a b l e} \mathbf{1}$ ). No complication of extraction such as wound dehiscence, nerve disturbance, or prolonged pain was registered, nor allergic reaction was experienced by participants.

In the present study, analysis of wound closure was performed by subtracting the wound diameter on Day 7 with wound diameter on Day 1 in buccolingual and mesiodistal directions (-Fig. 2). The mean values of wound diameter at different evaluation times are presented in -Table 2. The wound diameter in the test group decreased significantly in buccolingual, as well as mesiodistal direction compared with control group ( $p$-value $<0.05$ ).

Clinical features of wound healing were assessed on Day 7 by the use of Landry et al index (1988) (-Table 3). The frequency of healing scores experienced by subjects is presented in -Table 4 .
Correlation between healing score and the use of allocated gel was performed with Kendall's tau analysis and is presented in - Table 5. It can be seen that the use of $0.2 \% \mathrm{CHX}$ gel had significant correlation with healing scores ( $p$-value $<0.05$ ).

\section{Discussion}

Several local and general factors affect oral wound healing, such as trauma, thermal damage, ischemia, wound size and location, edema, and infection. Healing within the oral cavity is a critical aspect since it occurs in warm oral fluid containing millions of microorganisms. Therefore, infection frequently occurs leading to poor wound healing. The aim of the present study was to evaluate the effect of topical application of CHX gel twice a day performed by the patients on top of the postextraction wound site. Healing was defined

Table 2 Comparison of wound closure on Days 1 and 7 after tooth extraction

\begin{tabular}{|c|c|c|c|c|}
\hline & $\bar{X}(\mathrm{D} 1) \pm \mathrm{SD}(\mathrm{mm})$ & $\bar{X}(\mathrm{D} 7) \pm \mathrm{SD}(\mathrm{mm})$ & $\Delta \pm \mathrm{SD}(\mathrm{mm})$ & $p$-Value \\
\hline \multicolumn{5}{|c|}{ Buccolingual width } \\
\hline CHX $0.2 \%$ & $6.69 \pm 1.42$ & $2.41 \pm 1.13$ & $4.28 \pm 0.65$ & \multirow[t]{2}{*}{$0.0000000000637^{\mathrm{a}}$} \\
\hline Placebo & $6.06 \pm 1.81$ & $3.91 \pm 1.58$ & $2.15 \pm 0.60$ & \\
\hline \multicolumn{5}{|c|}{ Mesiodistal width } \\
\hline $\mathrm{CHX} 0.2 \%$ & $9.25 \pm 1.34$ & $5.00 \pm 1.22$ & $4.25 \pm 0.61$ & \multirow[t]{2}{*}{$0.0000000000144^{\mathrm{a}}$} \\
\hline Placebo & $8.40 \pm 1,85$ & $6.56 \pm 1.80$ & $1.84 \pm 0.72$ & \\
\hline
\end{tabular}

Abbreviations: CHX, chlorhexidine; SD, standard deviation.

Notes: $\bar{X}(D 1)=$ mean wound diameter on Day 1. $\bar{X}(D 7)=$ mean wound diameter on Day 7. $\Delta=$ wound diameter on D7 - D1.

${ }^{a}$ Significant difference.

Table 3 Wound healing index (Landry et al, 1988)

\begin{tabular}{|l|l|l|l|l|l|}
\hline Healing index & Tissue color & $\begin{array}{l}\text { Bleeding on } \\
\text { palpation }\end{array}$ & $\begin{array}{l}\text { Granulation } \\
\text { tissue }\end{array}$ & Incision margin & Suppuration \\
\hline $\begin{array}{l}\text { 1-very poor } \\
\text { Two or more signs } \\
\text { are present }\end{array}$ & $\geq 50 \%$ of red gingiva & Yes & Yes & $\begin{array}{l}\text { Not epithelized, with } \\
\text { loss of epithelium } \\
\text { beyond incision margin }\end{array}$ & Yes \\
\hline 2-poor & $\geq 50 \%$ of red gingiva & Yes & Yes & $\begin{array}{l}\text { Not epithelized, with exposed } \\
\text { connective tissue }\end{array}$ & No \\
\hline 3-good & 25-50\% of red gingiva & No & No & No exposed connective tissue & No \\
\hline 4-very good & $<25 \%$ of red gingiva & No & No & No exposed connective tissue & No \\
\hline 5-excellent & All pink tissues & No & No & No exposed connective tissue & No \\
\hline
\end{tabular}


Table 4 Results of wound healing index (Landry et al)

\begin{tabular}{|l|l|l|l|}
\hline & Healing index & N=32 \\
\cline { 3 - 4 } & & CHX & Placebo \\
\hline 1 & Very poor & 0 & 0 \\
\hline 2 & Poor & 0 & 0 \\
\hline 3 & Good & 5 & 5 \\
\hline 4 & Very good & 8 & 10 \\
\hline 5 & Excellent & 3 & 1 \\
\hline N & & 16 & 16 \\
\hline
\end{tabular}

Abbreviation: CHX, chlorhexidine.

as wound closure and standardized healing index evaluating clinical appearance.

The result of the present RCT showed that $0.2 \%$ CHX gel significantly improved wound healing at the clinical level. In the test group that was instructed to apply with $0.2 \% \mathrm{CHX}$ gel every 12 hours for 7 days after extraction, greater wound closure and better clinical healing were achieved as assessed by standardized healing index. This positive effect on clinical healing was related to the use of CHX gel as revealed by correlation analysis. In a meta-analysis study, Mínguez-Serra et al (2009) found that $0.2 \%$ CHX gel administered twice a day for 7 days would be the best option for preventing alveolar osteitis after extraction. ${ }^{21}$ In the form of mouth rinse, it was shown by Halabi et al (2018) that $0.12 \%$ CHX mouthwash was able to prevent alveolar osteitis in a population having risk of developing alveolar osteitis (previous surgical site infection, traumatic extraction, and tobacco smoking) after tooth extraction, whereas Hita-Iglesias et al (2008) found that CHX in the form of gel may decrease the incidence of alveolar osteitis after mandibular third molar extraction compared with mouth rinse (7.5 vs. $25 \%){ }^{20,22} \mathrm{~A}$ recent meta-analysis of $0.2 \%$ CHX gel application intra-alveolar showed that it was effective in preventing alveolar osteitis after third molar extraction. ${ }^{23}$ In this present study, we evaluate $\mathrm{CHX}$ gel considering that the method of administration of this gel has the main advantage of providing a greater bioavailability in the application area, and therefore, the medication has a more prolonged release. Furthermore, administration of CHX gel was self-administered by the participants, and not performed by operators, so this way of administration could be considered as home care treatment.

In addition, the majority of the studies evaluating the protective effect of CHX toward alveolar osteitis were conducted in surgery procedures with sutures to close the wounds. However, this present study was performed in patients having tooth extraction without sutures at the end of the procedures. Thus, the participants in this present study had open wounds, and no dressing were applied instead of the test gel or placebo. The results of this present study are in accordance with Palaia et al (2019) who investigated the effect of mouthwashes containing the combination of $\mathrm{CHX}$ and sodium hyaluronate, CHX alone and placebo in second intention wound healing after oral
Table 5 Correlation of healing scores and allocated gel

\begin{tabular}{|c|c|c|}
\hline & $p$-Value & $w$ \\
\hline \multicolumn{3}{|l|}{$\mathrm{CHX} 0.2 \%$} \\
\hline Width & \multirow[t]{2}{*}{$0.00000493^{a}$} & \multirow[t]{2}{*}{0.764} \\
\hline Healing index & & \\
\hline \multicolumn{3}{|l|}{ Placebo } \\
\hline Width & \multirow[t]{2}{*}{0.3232} & \multirow[t]{2}{*}{0.071} \\
\hline Healing index & & \\
\hline
\end{tabular}

Abbreviation: $\mathrm{CHX}$, chlorhexidine.

Note: $\mathrm{W}=$ Kendall's coefficient.

${ }^{\mathrm{a}}$ Significant difference.

biopsy with laser and without sutures. ${ }^{24} \mathrm{CHX}$ was proven to have accelerating effect on wound healing and can be recommended as good support or adjuvant therapy after surgical procedures. Furthermore, in a recent meta-analysis study, Armond et al (2017) suggested that the use of intraalveolar CHX gel after surgical removal of mandibular third molars reduces pain, edema, and trismus after the extraction of third molars. ${ }^{25}$

None of the participants of the CHX group in the present study experienced adverse events or hypersensitivity reactions after applying the gel. This could be the result of screening for CHX hypersensitivity before the commencement of the study that only individuals without allergic or hypersensitivity reactions to $\mathrm{CHX}$ could be included as participants. Nevertheless, several publications reported adverse events associated with prolonged use of CHX mouth rinse, ranged from mild to severe reactions including taste changes, tooth/tongue/staining, itching mouth, sore mouth, and increased calculus, while acute reactions had been reported as skin rash, nasal congestion, shortness of breath, swelling of face/lips/throat, nausea, swollen glands, diarrhea, abdominal pain, as well as anaphylactic reactions leading to death. ${ }^{17,26,27}$ One of the study by McCoy et al (2008) reported that these adverse events occurred in older adults with uncontrolled diabetes, while in the present study, the participants included were younger than 50 years and systemically healthy with ASA status $\mathrm{I}^{17}$ Therefore, careful monitoring of adverse reactions in patients using $\mathrm{CHX}$ is warranted particularly among those with multiple medical conditions and a history of allergies or breathing problems, and the clinicians must carefully and completely advise patients who use CHX, in any form, of possible side effects.

In the present study, smoker individuals were excluded because several studies demonstrated that smoking is associated with delayed healing, wound infection, and dehiscence. ${ }^{28-30}$ Nicotine in cigarettes has vasoconstriction effect, which may predispose to thrombotic microvascular occlusion and consequent tissue ischemia. According to Heng et al (2007), dry socket was found common among smokers after dental extractions as results of fibrinolytic activity and reduced alveolar blood supply. ${ }^{31}$ It should be remembered that this study was performed in a group of nonsmokers. 
Therefore, it is currently unknown to what extent the present results can be extrapolated to smokers.

A relevant action of $\mathrm{CHX}$ in promoting wound healing may be due to its ability to reduce the bacterial load on the wound. Bacterial infection is proven to have clinical significance in impairing wound healing since it interferes with the normal wound healing process by stimulating the body's defense through activation of inflammatory cells and mediators, which in turn destructs the granulation and the surrounding normal tissues. ${ }^{32}$ If inflammation persists, it will impair therapeutic intervention as well as tissue regeneration. ${ }^{33}$ Back to 1970, Lindhe et al demonstrated that one daily topical application of $2.0 \%$ to the teeth and gingiva was able to remove bacterial plaque and resolved gingival inflammation in dogs. ${ }^{34}$ Nevertheless, several studies on CHX have shown considerable contradiction on its effect on wound healing. Despite its strong bactericidal action, in vitro studies showed that CHX negatively affected fibroblast and keratinocyte proliferation in a dose-dependent manner, ${ }^{35-37}$ while in vivo studies demonstrated the opposites. ${ }^{38-40}$ These contrary results might be due to the different cellular and molecular interactions occurring in vitro and in vivo. In vivo, CHX in the form of positively charged bisbiguanide can bind to different negatively charged sites, including mucous membranes, salivary pellicle on teeth, and titanium surfaces, as well as several components of the biofilm on the tooth surfaces, for example, bacteria, extracellular polysaccharides, and glycoproteins. ${ }^{41,42}$ Therefore, the remaining amount of CHX molecules available to bind to and harm host cells in the wound is significantly reduced. ${ }^{43}$

Third molar surgery study design is usually the most validated study model to evaluate the effects of antimicrobial products on the postoperative period after tooth extraction, ${ }^{20-23,44}$ but since the administration of $\mathrm{CHX}$ gel in those trial was more complicated than what was performed in this present study, we showed that CHX gel could also be administered by the patients themselves without having surgeon applying the gel into the postextraction socket. The ease and convenient way of this administration may be considered as home care for patients who underwent noncomplicated extractions.

A limitation of the present study is the relatively short period of early wound healing and that we only registered wound healing, while the severity of pain experienced by the participants was not recorded.

\section{Conclusion}

In conclusion, under limitation of the present study, it is confirmed that CHX gel has the beneficial effect in enhancing wound healing after nonsurgery tooth extraction and may be suggested as adjuvant treatment and home care. It merits further studies to evaluate the effect of CHX with the evaluation of clinical and radiographic examination after tooth extraction.

Funding

None.
Conflict of Interest

None declared.

Acknowledgments

We would like to thank Head of Pharmacy Technology Laboratory, Faculty of Math and Science, Universitas Padjadjaran, Jalan Raya Bandung - Sumedang KM 21, Jatinangor for preparing the placebo gel.

\section{References}

1 Adeyemo WL, Ladeinde AL, Ogunlewe MO. Clinical evaluation of post-extraction site wound healing. J Contemp Dent Pract 2006;7 (03):40-49

2 Al-Khateeb TH, Alnahar A. Pain experience after simple tooth extraction. J Oral Maxillofac Surg 2008;66(05):911-917

3 Tong DC, Al-Hassiny HH, Ain AB, Broadbent JM. Post-operative complications following dental extractions at the School of Dentistry, University of Otago. N Z Dent J 2014;110(02):51-55

4 Nooh N, Graves DT. Healing is delayed in oral compared to dermal excisional wounds. J Periodontol 2003;74(02):242-246

5 Anderson K, Hamm RL. Factors that impair wound healing. J Am Coll Clin Wound Spec 2014;4(04):84-91

6 Sekhar A, Basheer SA, Nair A. Microbiological evaluation of bacterial plaque in suture material used post-extraction. J Crit Rev 2020;7(19):10164-10173

7 Ford SJ. The importance and provision of oral hygiene in surgical patients. Int J Surg 2008;6(05):418-419

8 Ripari F, Cera A, Freda M, Zumbo G, Zara F, Vozza I. Tea tree oil versus chlorhexidine mouthwash in treatment of gingivitis: a pilot randomized, double blinded clinical trial. Eur J Dent 2020;14 (01):55-62

9 Fry DE. Topical antimicrobials and the open surgical wound. Surg Infect (Larchmt) 2016;17(05):520-524

10 Sarabahi S. Recent advances in topical wound care. Indian J Plast Surg 2012;45(02):379-387

11 Cooper R. A review of the evidence for the use of topical antimicrobial agents in wound care. World Wide Wounds 2004;1:1-8

12 Varoni E, Tarce M, Lodi G, Carrassi A. Chlorhexidine (CHX) in dentistry: state of the art. Minerva Stomatol 2012;61(09): 399-419

13 Fiorillo L. Chlorhexidine gel use in the oral district: a systematic review. Gels 2019;5(02):31

14 Brecx M, Netuschil L, Reichert B, Schreil G. Efficacy of Listerine, Meridol and chlorhexidine mouthrinses on plaque, gingivitis and plaque bacteria vitality. J Clin Periodontol 1990;17(05):292-297

15 Van Strydonck DA, Slot DE, Van der Velden U, Van der Weijden F. Effect of a chlorhexidine mouthrinse on plaque, gingival inflammation and staining in gingivitis patients: a systematic review. J Clin Periodontol 2012;39(11):1042-1055

16 Supranoto SC, Slot DE, Addy M, Van der Weijden GA. The effect of chlorhexidine dentifrice or gel versus chlorhexidine mouthwash on plaque, gingivitis, bleeding and tooth discoloration: a systematic review. Int J Dent Hyg 2015;13(02):83-92

17 McCoy LC, Wehler CJ, Rich SE, Garcia RI, Miller DR, Jones JA. Adverse events associated with chlorhexidine use: results from the Department of Veterans Affairs Dental Diabetes Study. J Am Dent Assoc 2008;139(02):178-183

18 Kumar MA, Gheena S. Incidence of dry socket after third molar extraction. J Pharm Sci Res. 2015;7(07):451

19 Hammad HM, Hammad MM, Abdelhadi IN, Khalifeh MS. Effects of topically applied agents on intra-oral wound healing in a rat model: a clinical and histomorphometric study. Int J Dent Hyg 2011;9(01):9-16

20 Halabi D, Escobar J, Alvarado C, Martinez N, Muñoz C. Chlorhexidine for prevention of alveolar osteitis: a randomised clinical trial. J Appl Oral Sci 2018;26:e20170245 
21 Landry RG, Turnbull RS, Howley T. Effectiveness of benzydamine $\mathrm{HCl}$ in the treatment of periodontal postsurgical patients. Research in Clinic Forums 1988;10:105-118

22 Hita-Iglesias P, Torres-Lagares D, Flores-Ruiz R, Magallanes-Abad $\mathrm{N}$, Basallote-Gonzalez M, Gutierrez-Perez JL. Effectiveness of chlorhexidine gel versus chlorhexidine rinse in reducing alveolar osteitis in mandibular third molar surgery. J Oral Maxillofac Surg 2008;66(03):441-445

23 Zhou J, Hu B, Liu Y, Yang Z, Song J. The efficacy of intra-alveolar $0.2 \%$ chlorhexidine gel on alveolar osteitis: a meta-analysis. Oral Dis 2017;23(05):598-608

24 Palaia G, Tenore G, Tribolati L, et al. Evaluation of wound healing and postoperative pain after oral mucosa laser biopsy with the aid of compound with chlorhexidine and sodium hyaluronate: a randomized double blind clinical trial. Clin Oral Investig 2019; 23(08):3141-3151

25 Armond AC, Milani LM, Fonseca JD, de Castro Martins C, Falci SG. Does the use of intra-alveolar chlorhexidine gel reduces the rate of alveolar osteitis, pain, edema and trismus after the extraction of lower third molars? A meta analysis. J Oral Maxillofac Surg Med Pathol 2017;29(06):491-498

26 Pemberton MN, Gibson J. Chlorhexidine and hypersensitivity reactions in dentistry. Br Dent J 2012;213(11):547-550

27 Nakonechna A, Dore P, Dixon T, et al. Immediate hypersensitivity to chlorhexidine is increasingly recognised in the United Kingdom. Allergol Immunopathol (Madr) 2014;42(01):44-49

28 Sørensen LT. Wound healing and infection in surgery: the pathophysiological impact of smoking, smoking cessation, and nicotine replacement therapy: a systematic review. Ann Surg 2012;255 (06):1069-1079

29 Abu Younis MH, Abu Hantash RO. Dry socket: frequency, clinical picture, and risk factors in a Palestinian dental teaching center. Open Dent J 2011;5:7-12

30 Kean J. The effects of smoking on the wound healing process. J Wound Care 2010;19(01):5-8

31 Heng CK, Badner VM, Clemens DL, Mercer LT, Mercer DW. The relationship of cigarette smoking to postoperative complications from dental extractions among female inmates. Oral Surg Oral Med Oral Pathol Oral Radiol Endod 2007;104(06): 757-762
32 Guo S, Dipietro LA. Factors affecting wound healing. J Dent Res 2010;89(03):219-229

33 Shukla SK, Sharma AK, Gupta V, Yashavarddhan MH. Pharmacological control of inflammation in wound healing. J Tissue Viability 2019;28(04):218-222

34 Lindhe J, Hamp SE, Löe H, Schiøtt CR. Influence of topical application of chlorhexidine on chronic gingivitis and gingival wound healing in the dog. Scand J Dent Res 1970;78(06):471-478

35 Hidalgo E, Dominguez C. Mechanisms underlying chlorhexidineinduced cytotoxicity. Toxicol In Vitro 2001;15(4-5):271-276

36 Balloni S, Locci P, Lumare A, Marinucci L. Cytotoxicity of three commercial mouthrinses on extracellular matrix metabolism and human gingival cell behaviour. Toxicol In Vitro 2016;34:88-96

37 Wyganowska-Swiatkowska M, Urbaniak P, Szkaradkiewicz A, Jankun J, Kotwicka M. Effects of chlorhexidine, essential oils and herbal medicines (Salvia, Chamomile, Calendula) on human fibroblast in vitro. Cent Eur J Immunol 2016;41(02):125-131

38 Kozlovsky A, Artzi Z, Hirshberg A, Israeli-Tobias C, Reich L. Effect of local antimicrobial agents on excisional palatal wound healing: a clinical and histomorphometric study in rats. J Clin Periodontol 2007;34(02):164-171

39 Paolantonio M, Perinetti G, D'Ercole S, et al. Internal decontamination of dental implants: an in vivo randomized microbiologic 6month trial on the effects of a chlorhexidine gel. J Periodontol 2008;79(08):1419-1425

40 Jiang B, Zhang G, Brey EM. Dual delivery of chlorhexidine and platelet-derived growth factor-BB for enhanced wound healing and infection control. Acta Biomater 2013;9(02):4976-4984

41 Kozlovsky A, Artzi Z, Moses O, Kamin-Belsky N, Greenstein RB. Interaction of chlorhexidine with smooth and rough types of titanium surfaces. J Periodontol 2006;77(07):1194-1200

42 Hjeljord LG, Rölla G, Bonesvoll P. Chlorhexidine-protein interactions. J Periodontal Res Suppl 1973;12:11-16

43 Jenkins S, Addy M, Wade W. The mechanism of action of chlorhexidine. A study of plaque growth on enamel inserts in vivo.J Clin Periodontol 1988;15(07):415-424

44 Mínguez-Serra MP, Salort-Llorca C, Silvestre-Donat FJ. Chlorhexidine in the prevention of dry socket: effectiveness of different dosage forms and regimens. Med Oral Patol Oral Cir Bucal 2009;14 (09):e445-e449 